\title{
Partitioning Conformational Intermediates Between Competing Refolding and Aggregation Pathways: Insights into Transthyretin Amyloid Disease ${ }^{\dagger}$
}

\author{
R. Luke Wiseman, Evan T. Powers, and Jeffery W. Kelly ${ }^{\star}$ \\ Department of Chemistry and the Skaggs Institute of Chemical Biology, The Scripps Research \\ Institute, 10550 N. Torrey Pines Rd., BCC 265, La Jolla, CA 92037
}

\begin{abstract}
Amyloid diseases are caused by the aberrant assembly of a protein in the extracellular space. Folded proteins are not amyloidogenic, however the native state is generally in equilibrium with a minor population of unfolded or partially folded aggregation-competent conformers outside of the cell. Understanding how the partially unfolded conformers kinetically partition between the competing refolding and aggregation pathways provides insight into how misfolding, which occurs continuously, becomes pathogenic. Towards this end we have previously studied the amyloidogenicity of transthyretin (TTR), a human $\beta$-sheet rich homotetrameric protein that must undergo rate-limiting tetramer dissociation and partial monomer unfolding to misassemble into amyloid and other aggregates. We demonstrate herein that TTR homotetramers reassemble by an unusual monomer-dimer-trimer-tetramer (MDRT) pathway. Therefore, the rate of every step in the reassembly pathway is dependent on the concentration of folded TTR monomer. Partitioning soluble TTR monomers between the reassembly pathway and the aggregation pathway should therefore depend on the relative concentrations of aggregates and assembly intermediates. Aggregate clearance is envisioned to play an important role in the partitioning of protein in vivo, where partitioning to the aggregation pathway becomes increasingly favorable under conditions where the concentration of aggregates is increased because aggregate clearance is slow relative to the rate of aggregation. This shift from efficient to inefficient aggregate clearance could occur with aging, offering an explanation for the age-associated nature of these neurodegenerative diseases.
\end{abstract}

\begin{abstract}
Amyloid diseases are characterized by the extracellular misassembly of a given secreted protein into cross $\beta$-sheet structures of various morphologies (1-3). Over 20 non-homologous human proteins are known to be amyloidogenic. Genetic, biochemical, and pathological evidence suggest that the process of amyloidogenesis causes disease. Both natively unfolded proteins and those that adopt well-defined conformations can be amyloidogenic. The latter group must adopt partially unfolded states in order to aggregate (4). Moreover, the partially unfolded conformations must be kinetically partitioned away from the refolding pathway to the aggregation pathway. Kinetic partitioning between reconstitution and aggregation has been studied for several proteins. Biophysical and cell biological studies on the folding of the P22 tailspike protein reveal folding intermediates that are capable of partitioning into either the aggregation or reassembly pathways (5). Recent studies on acylphosphatase variants demonstrate that kinetic partitioning between the folding and aggregation pathways is influenced by the hydrophobicity and $\beta$-sheet propensity of the mutated residue in the
\end{abstract}

\footnotetext{
${ }^{\dagger}$ This work was supported in part by NIH Grants DK46335, the Skaggs Institute for Chemical Biology, the Lita Annenberg Hazen Foundation, the Norton B. Gilula Fellowship (RLW), and the Fletcher Jones Foundation Fellowship (RLW).

* Corresponding author: jkelly@ scripps.edu; Phone: (858) 784-9601; Fax: (858) 784-9610.
} 
acylphosphatase sequence in aqueous organic solvents, where both pathways are accessible (6). Given that almost all secreted folded proteins can sample partially unfolded states to an extent governed by the folding energetics, it is important to understand how this process becomes pathogenic in certain individuals. It seems clear that the aggregation and native refolding pathways must be characterized in vitro in order to begin to understand the kinetic partitioning between these pathways in vivo, as it relates to amyloid disease.

The native homotetrameric structure of transthyretin (TTR) is required for its function, which includes the transport of both the holo-retinol binding protein and the small molecule hormone thyroxine $\left(\mathrm{T}_{4}\right)$ in the blood and cerebral spinal fluid, utilizing separate binding sites made up of more than one subunit (7). TTR is known to spontaneously dissociate, albeit slowly, and sample a partially unfolded state that allows its misassembly into a spectrum of aggregates, including amyloid fibrils, a process causatively linked to several human diseases $(1,8)$. Deposits of wild-type TTR appear to cause Senile Systemic Amyloidosis (SSA), affecting at least $10 \%$ of the population over age 80 , whereas the amyloidogenicity of one of over 100 TTR point mutations leads to the familial TTR amyloid diseases including Familial Amyloid Polyneuropathy (FAP), Familial Amyloid Cardiomyopathy (FAC), and central nervous system selective amyloidosis (CNSA) (9-19)

TTR aggregation commences by rate-limiting dissociation of the native tetramer followed by partial unfolding of the monomeric subunit to an aggregation competent conformation(s), hereafter referred to as the amyloidogenic intermediate $(4,8,20-22)$. The aggregation of the amyloidogenic intermediate proceeds via a downhill polymerization mechanism under acidic conditions, and also possibly under physiological conditions (23). In this process, the bimolecular reaction between amyloidogenic intermediates proceeds at the same rate as the addition of an amyloidogenic intermediate to larger aggregates, with both processes being energetically favorable. Unlike a nucleated polymerization reaction, which requires the formation of a high-energy oligomeric nucleus before aggregation becomes spontaneous and wherein preformed oligomers, commonly referred to as seeds, accelerate the aggregation reaction, TTR aggregation does not require the formation of a high energy nucleus and is not accelerated by seeds under denaturing conditions (low $\mathrm{pH}$ ) (23). The rate of a downhill aggregation process is proportional to the concentration of TTR amyloidogenic intermediate in solution. Under denaturing conditions where TTR tetramer dissociation is largely irreversible and partial unfolding of the monomer is favorable (e.g. $\mathrm{pH} 4-5$ or in $50 \%$ methanol), the rate of monomeric amyloidogenic intermediate formation, and therefore the aggregation rate, is dependent solely on the rate of tetramer dissociation (24).

The aggregation rate of TTR in vivo is dependent not only on the rate of tetramer dissociation, but also on the kinetic partitioning of the amyloidogenic intermediate between the aggregation and competing reassembly pathways. Understanding the variables governing this kinetic partitioning under physiological conditions will lead to a better understanding of the scenarios resulting in extracellular TTR deposition. Such an understanding, however, requires knowledge of the mechanism by which the native tetramer reassembles, which is the focus of the study herein.

The TTR homotetrameric structure possesses 2,2,2 symmetry and is arranged as a dimer of dimers (Figure 1A-C) (7). Given the two types of dimer interfaces and the symmetry exhibited (Figure 1 B,C), the TTR tetramer could assemble through a variety of different pathways. The assembly mechanism of a number of homotetrameric proteins has been elucidated, and in all cases, reassembly is postulated to occur by a monomer (M) - dimer (D) - tetramer (T) (MDT) pathway (25-33). In an MDT mechanism, two monomeric subunits combine to form a dimer, which subsequently interacts with another dimer, affording a tetramer. TTR could also assemble by sequential addition of monomers through a monomer (M) - dimer (D) - trimer 
(R) - tetramer (T) (MDRT) pathway. Although no homotetramers assemble by this mechanism to our knowledge, the MDRT mechanism must be considered as a possibility for the reassembly of TTR homotetramers. The MDRT mechanism is distinct from the MDT mechanism because the rate of every step along the MDRT reassembly pathway is dependent on the concentration of monomers in solution. Since these mechanisms have different monomer concentration dependencies, they can be distinguished by evaluating the reassembly kinetics of TTR monomer over a wide concentration range. The concentration dependent TTR reassembly kinetics were fit to a number of potential pathways, revealing that TTR homotetramers reassemble by the unusual MDRT pathway (although the existence of fast reorganization steps integrated into the MDRT pathway could not be ruled out). The rate constants defined by this MDRT mechanism are such that under normal conditions, monomeric TTR partitions effectively into the tetramer reconstitution pathway, and does not aggregate appreciably. However, under conditions where soluble aggregates exist at appreciable concentrations, the partitioning of protein into the aggregate state can occur more favorably as the concentration of soluble aggregates increases. This balance between reassembly and aggregation could influence the onset of amyloid disease.

\section{Materials and Methods}

\section{Expression of wild-type and flag-tag wild-type TTR}

Wild type and flag tag wild-type TTR were prepared as previously described (34). Plasmids containing either wild-type TTR (pmmHa) or flag-tag wild type TTR (pet-29a) were transformed into competent Epicurian Gold E. coli cells. Cells were grown in one liter cultures of Luria-Bertani media containing $100 \mu \mathrm{g} / \mathrm{mL}$ ampicillin (wild-type TTR) or $150 \mu \mathrm{g} / \mathrm{mL}$ kanamycin (flag-tag wild-type TTR). The cells were grown until the $\mathrm{OD}_{600 \mathrm{~nm}}$ was $>0.8$. Protein expression was then induced by the addition of $1 \mathrm{mM}$ IPTG to the media. The cultures were incubated at $37^{\circ} \mathrm{C}$ overnight. The cells were pelleted by centrifugation ( 8 min; 10,000 RPM) and lysed with three rounds of sonication at $4{ }^{\circ} \mathrm{C}$. The lysate was then collected by centrifugation (14,000 RPM; $30 \mathrm{~min}$ ) and treated with $50 \%$ (w/v) ammonium sulfate. The solution was centrifuged (14,000 RPM; $30 \mathrm{~min})$ and the lysate was collected and treated with $90 \%$ ammonium sulfate. The precipitate from the $90 \%$ ammonium sulfate treatment was collected and dissolved in $25 \mathrm{mM}$ Tris buffer (pH 8.0), with $1 \mathrm{mM}$ EDTA. The solution was dialyzed overnight into $25 \mathrm{mM}$ Tris $\mathrm{pH} 8.0,1 \mathrm{mM}$ EDTA at $4{ }^{\circ} \mathrm{C}$. Protein was then isolated from a Source $\mathrm{Q}$ anion exchange column (Pharmacia) running either a $5-350 \mathrm{mM} \mathrm{NaCl}$ gradient (wild-type) or a $200-450 \mathrm{mM} \mathrm{NaCl}$ gradient (flag tag wild-type). The final protein product was then purified by a Superdex 75 gel filtration column (Pharmacia) to remove any aggregates (buffer: $10 \mathrm{mM}$ sodium phosphate buffer (pH 7.2) with $100 \mathrm{mM} \mathrm{KCl}$ and $1 \mathrm{mM}$ EDTA). The concentration of wild-type and flag-tag wild-type TTR were determined by UV absorbance using the protein extinction coefficients (wild-type - $18259 \mathrm{M}^{-1} \mathrm{~cm}^{-1}$; flag-tag wild-type TTR - $21056 \mathrm{M}^{-1} \mathrm{sec}^{-1}$; monomer).

\section{Denaturation of wild-type and flag-tag wild-type TTR for reassembly analysis}

Wild-type and flag-tag wild-type TTR were concentrated to fifty times the desired concentration of protein then denatured by a 1:5 dilution of protein into $10 \mathrm{M}$ urea in $50 \mathrm{mM}$ sodium phosphate buffer ( $\mathrm{pH}$ 7.2) with $100 \mathrm{mM} \mathrm{KCl}$ and $1 \mathrm{mM}$ EDTA. The samples were stored at $4{ }^{\circ} \mathrm{C}$ for 96 hours, demonstrating complete denaturation by measuring the intrinsic Trp fluorescence of TTR on an Aviv ATF4 Spectrofluorometer (excitation $295 \mathrm{~nm}$ bandwidth $2.00 \mathrm{~nm}$, emission $310-410 \mathrm{~nm}$ bandwidth $6.00 \mathrm{~nm}$ ).

\section{Small molecule TTR reassembly assay}

Wild-type TTR denatured as described above, was diluted 1:10 into a buffer of $50 \mathrm{mM}$ sodium phosphate buffer (pH 7.2) with $100 \mathrm{mM} \mathrm{KCl} 1 \mathrm{mM}$ EDTA containing a 10-fold molar excess 
of $\mathbf{1}$ (as compared to the tetrameric concentration of TTR) using an Aviv stopped flow apparatus with a $10 \mathrm{msec}$ mixing time (final urea concentration $=0.8 \mathrm{M}$; final TTR concentration $=$ as indicated). The assembly of the TTR tetramers was monitored by measuring the continuous fluorescence signal at $515 \mathrm{~nm}$ (bandwidth $10.00 \mathrm{~nm}$ ) with excitation at $320 \mathrm{~nm}$ (bandwidth $2.00 \mathrm{~nm})$.

\section{Glutaraldehyde crosslinking TTR reassembly assay}

Wild-type TTR, denatured as described above, was diluted into a stirring solution of $50 \mathrm{mM}$ sodium phosphate buffer ( $\mathrm{pH} 7.2)$ with $100 \mathrm{mM} \mathrm{KCl}$ and $1 \mathrm{mM}$ EDTA with or without a 10fold molar excess of 1 . Glutaraldehyde $(25 \%, 5 \mu \mathrm{L})$ was added to a $50 \mu \mathrm{L}$ aliquot of the reaction solution at the indicated time. The glutaraldehyde reaction was allowed to proceed for four minutes, when the reaction was stopped by the addition of $5 \mu \mathrm{L}$ of $7 \%$ sodium borohydride in $0.1 \mathrm{M}$ sodium hydroxide. The crosslinked samples were boiled in reducing gel loading buffer for $5 \mathrm{~min}$. The samples were then run on a $12 \%$ SDS-PAGE gel, and the gels were subjected to silver staining. The density of the gel bands representing the various TTR species were examined using the program Scion Image.

\section{Flag-tag wild-type TTR subunit incorporation assay for evaluating TTR reassembly kinetics}

Wild-type TTR, denatured as described above, was diluted into a stirring solution of $50 \mathrm{mM}$ sodium phosphate buffer (pH 7.2) with $100 \mathrm{mM} \mathrm{KCl}$ and $1 \mathrm{mM}$ EDTA with or without a 10fold molar excess of $\mathbf{1}$. At the indicated time points, denatured flag-tag wild-type TTR was simultaneously diluted 1:10 into the refolding buffer and added to the wild-type reassembly reaction. The reaction was then allowed to proceed for 10 minutes allowing all of the protein to assemble into tetramers. The samples were then injected onto a SMART system (Pharmacia) using a Mono Q PC/1.65 anion exchange column (Pharmacia), separating the various tetramers of defined stoichiometry by increasing the concentration of sodium chloride from $240-420$ $\mathrm{mM}$ in $25 \mathrm{mM}$ Tris $\mathrm{pH} 8.01 \mathrm{mM}$ EDTA. The amount of each diastereomeric TTR tetramer was quantified by integration of the protein peaks using the SMART Manager protocol (Pharmacia). The fraction of each peak follows from the integration of a given peak divided by the total integration of all peaks.

\section{Fitting analysis of the concentration dependent TTR reassembly time course data}

Global fitting of the concentration dependent TTR reassembly time courses was performed on a personal computer with dual AMD Athlon 2200 MP processors using Mathematica 4.2 (Wolfram Research) for Windows XP. The concentration dependent reassembly time courses resulting in TTR tetramer formation were globally fit to Mechanisms C, D, and E (utilizing the rate equations defined in the text) by varying the rate constants associated with the reassembly mechanisms to minimize the sum of squares difference between the predicted and experimental results (variants of Mechanism E, including conformational rearrangements or alternative dimer formation, were also evaluated as described in the Supporting Information). The global fitting was performed 45 times with random starting values for the variable rate constants associated with the specific mechanism of reassembly to ensure that the best fits represent the best global fit of the experimental data. It was generally observed that the best global fits of the experimental data corresponded to a single set of reaction parameters. The predicted reassembly kinetics were then plotted against the experimental results using Kaleidograph. 


\section{Results}

\section{Reassembly of TTR homotetramers can be accurately measured by a small molecule ligand binding assay}

Small molecule ligand binding assay-Previous studies demonstrate that denatured wild-type TTR reassembles to a functional homotetramer state within a few minutes $(7.2 \mu \mathrm{M}$, monomer) $(10,35,36)$. Established methodology including analytical gel filtration and analytical ultracentrifugation do not provide the time resolution required to follow this reassembly timecourse. Therefore, a stopped-flow assay was developed to measure the rate of TTR tetramer formation from an ensemble of denatured monomers, taking advantage of the selective binding-induced fluorescence exhibited by select small molecules that recognize the $\mathrm{T}_{4}$ binding sites in the native tetramer (Figure 2A). Small molecule $\mathbf{1}$ (Figure 3A) is ideal for monitoring the kinetics of tetramer formation in that $\mathbf{1}$ only fluorescences when it binds to the TTR tetramer non-cooperatively $\left(\mathrm{K}_{1}=\mathrm{K}_{2}=2 \times 10^{8} \mathrm{M}^{-1}\right.$,Figure 3A) (35). The rapid highaffinity binding of $\mathbf{1}$ to the native state allows TTR tetramer formation to be measured over a wide range of TTR concentrations. We demonstrate below that $\mathbf{1}$ does not influence TTR reassembly kinetics using crosslinking and subunit incorporation methodology.

The rate of TTR homotetramer reassembly was followed by rapidly diluting denatured (8.0 M urea) wild-type TTR 10-fold with refolding buffer containing a 10-fold molar excess of $\mathbf{1}$ (relative to the concentration of the TTR tetramer following reconstitution; Figure 2A) utilizing an Aviv stopped flow mixer. The environment-dependent fluorescence (excitation: $320 \mathrm{~nm}$; emission: $515 \mathrm{~nm}$ ), of $\mathbf{1}$ was employed to follow the tetramer formation time course over a 50fold TTR concentration range $(0.72-36 \mu \mathrm{M}$ monomer; Figure $3 \mathrm{~B})$ inclusive of the physiological concentration range exhibited by TTR $(8-28 \mu \mathrm{M}$; based on the monomer) in human serum. Control experiments evaluating the fluorescence signal of $\mathbf{1}$ bound to nondenatured TTR tetramers added to the reassembly buffer ( $0.8 \mathrm{M}$ urea, 10 -fold molar excess of 1 relative to the TTR tetramer) reveal that $>90 \%$ of TTR in $8.0 \mathrm{M}$ urea successfully reassembles to tetramers upon dilution (data not shown). These control experiments also demonstrate that the fluorescence reassembly kinetics are not complicated by photobleaching.

At the lower TTR concentrations ( $1.08 \mu \mathrm{M}$, monomer; Figure 3B), tetramer reassembly was complete within $30 \mathrm{~min}$ (Figure 3B, inset). Increasing the TTR concentration dramatically increased the rate of reassembly, with the reassembly reaction being complete on the timescale of seconds for the highest protein concentrations studied ( $36 \mu \mathrm{M}$ monomer; Figure 3B). The concentration dependent reassembly time courses were found to be reproducible when evaluated in triplicate - the standard deviation of the time to $50 \%$ completion $\left(\mathrm{t}_{50}\right)$ was \pm 10 $\%$ for all concentrations. In order to demonstrate that compound $\mathbf{1}$ does not influence the rate of TTR reassembly, the reassembly time course was evaluated by two additional independent methods.

\section{Measuring TTR tetramer reassembly by glutaraldehyde crosslinking demonstrates that the binding of 1 does not influence the kinetics of reassembly}

- Glutaraldehyde crosslinking has been used to evaluate the quaternary structure of TTR under a variety of denaturing conditions (20). To follow the reassembly of TTR by glutaraldehyde crosslinking, denatured wild-type TTR (14.4 or $43.2 \mu \mathrm{M}$ monomer; $8.0 \mathrm{M}$ urea) was diluted 10 -fold into refolding buffer (Figure 2B). At the indicated time points, aliquots of the reaction solution were removed and treated with glutaraldehyde utilizing a previously described crosslinking protocol $(20,37)$. TTR reassembly was monitored by the appearance of tetramer on an SDS-PAGE gel (Figure 4A). Glutaraldehyde crosslinking of the TTR tetramer is not an efficient process; therefore, after completion of the reassembly reaction, only $60-70 \%$ of the protein appears to be tetrameric (Figure 4A). Dimer bands are also observed, but it is difficult to determine if these bands are populated intermediates along the reassembly pathway or 
artifacts from the inefficient crosslinking of TTR tetramers. As a consequence, only the tetramer band is utilized as a measure of the completeness of the reassembly reaction. Furthermore, the glutaraldehyde crosslinking procedure was limited to protein concentrations between $1.44-4.32 \mu \mathrm{M}$ (monomer), where the crosslinking is fast relative to the reassembly kinetics.

TTR (4.32 $\mu \mathrm{M}$, monomer) reassembly followed by glutaraldehyde crosslinking occurs on the same time-scale as reassembly measured by the binding and fluorescence of $\mathbf{1}$ (Figure 4B). Of equal importance, the reassembly rate of TTR (1.44 or $4.32 \mu \mathrm{M}$, monomer), as measured by glutaraldehyde crosslinking, is the same in the presence and absence of a 10-fold molar excess (relative to the TTR tetramer) of $\mathbf{1}(3.6 \mu \mathrm{M}$ or $10.8 \mu \mathrm{M}$, respectively), demonstrating that $\mathbf{1}$ does not affect the reassembly kinetics of TTR (Figure 4C (1.44 $\mu \mathrm{M}), 4 \mathrm{D}(4.32 \mu \mathrm{M})$ ). The nearly identical kinetics revealed by glutaraldehyde crosslinking and the stopped-flow fluorescence assay also reveal that $\mathbf{1}$ does not bind to intermediates along the reassembly pathway.

\section{TTR tetramer reassembly time course followed by labeled subunit incorporation also demonstrates that the binding of 1 does not influence the TTR homotetramer reassembly kinetics-To further demonstrate that $\mathbf{1}$ does not affect the} kinetics of the TTR reassembly process, an assay was developed that takes advantage of the incorporation of flag-tagged wild-type TTR subunits into reassembling TTR tetramers (Figure 2C) $(38,39)$. In this reconstitution assay, denatured wild-type TTR (43.2 $\mu \mathrm{M}$, monomer; 8.0 $\mathrm{M}$ urea) was diluted 10-fold into refolding buffer. At various time points ( $\left.\mathrm{t}_{\text {wild-type }}\right)$ in the wildtype reassembly process, denatured wild-type TTR bearing an N-terminal acidic flag-tag (flagtag wild-type TTR; 43.2 $\mu \mathrm{M}$, monomer; 8.0 M urea) was simultaneously diluted 10-fold into a refolding buffer (final urea concentration remains $0.8 \mathrm{M}$; total protein concentration remains $4.32 \mu \mathrm{M}$, monomer) and added to the wild-type reassembly reaction. The reassembly reaction was then allowed to proceed to completion and the resulting heterotetramer distribution was evaluated by anion exchange chromatography, providing a chromatographic approach to follow the reassembly time course (Figure 5A) (38). The extent of wild-type TTR reassembly before the addition of flag-tag subunits can be determined from the distribution of tetramers 2-6 as a function of $\mathrm{t}_{\text {wild-type }}$ (tetramer $\mathbf{2}=(\text { wild-type TTR })_{4}$; tetramer $\mathbf{3}=(\text { wild-type TTR })_{3}$ (flag-tag wild-type TTR $)_{1}$; tetramer $\mathbf{4}$ = (wild-type TTR $)_{2}$ (flag-tag wild-type TTR $)_{2}$; tetramer $\mathbf{5}=(\text { wild-type TTR })_{1}(\text { flag-tag wild-type TTR })_{3}$; tetramer $\left.\mathbf{6}=(\text { flag-tag wild-type TTR })_{4}\right)$. At early time points, before significant reassembly to wild-type tetramers occurs, the addition of flag-tag wild-type subunits will result in a near statistical (1:4:6:4:1) distribution of tetramers 2-6 (Figure 5A - black trace, and 5B). Allowing the wild-type TTR reassembly reaction to proceed longer and therefore to a greater extent before addition of the flag-tag wild-type subunits will bias the distribution of wild-type and flag-tag wild-type TTR subunits towards homotetramers (tetramers 2 and 6; Figure 5A - green and red traces, and 5B - orange and black traces) to the exclusion of tetramers 3-5 (Figure 5B-3-blue trace, $\mathbf{4}$ - green trace, and $\mathbf{5}$ - red trace).

The TTR tetramer reassembly kinetics as measured by subunit incorporation are very similar to those measured by glutaraldehyde crosslinking and the fluorescence ligand binding assay (Figure 5C). Furthermore, the rate of tetramer reassembly as ascertained by subunit incorporation does not change in the presence of a 10-fold molar excess of $\mathbf{1}$ (relative to the tetramer), demonstrating that the small molecule does not hasten the reassembly of TTR homotetramers (Figure 5D). Moreover, the nearly identical time courses displayed by subunit incorporation and the fluorescent ligand binding assay demonstrate that $\mathbf{1}$ does not appear to bind to reassembly intermediates. The glutaraldehyde crosslinking results taken together with the labeled subunit incorporation data demonstrate that the ligand binding fluorescence assay accurately measures TTR reassembly kinetics. 


\section{Fitting the concentration dependent TTR reassembly rate data indicates that TTR reassembles by an unusual mechanism}

In order to evaluate the mechanism of homotetramer reassembly, potential mechanistic pathways must be systematically evaluated. Towards that end, we explore the concentration dependent behavior of different reassembly pathways to discern the best fit between experimental data and the expected data generated from mathematical models. TTR tetramers could reassemble by a number of mechanisms. The majority of these mechanisms have different TTR monomer concentration dependencies; therefore globally fitting the TTR reassembly data over a 50-fold concentration range would allow for the determination of the reaction pathway that best fits the experimental data. The mechanisms to be examined are shown below.

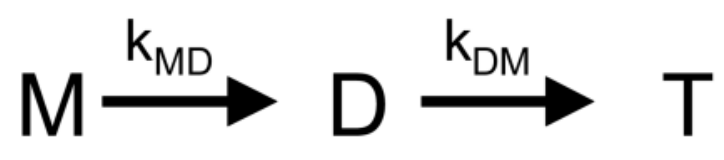

Mechanism A.

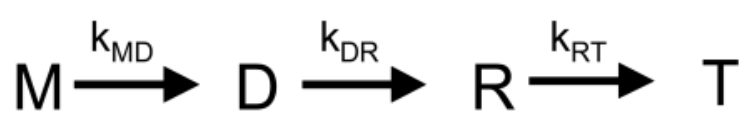

Mechanism B.

The first mechanisms examined will be the irreversible MDT and MDRT pathways

(Mechanisms A and B, respectively). These represent the simplest possible pathways for TTR tetramer reconstitution from monomers.

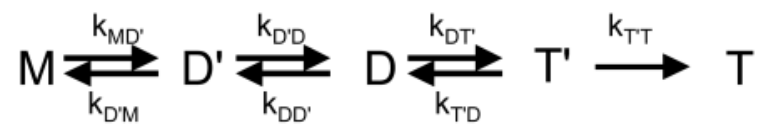

Mechanism C.

The next mechanism explored will be the MDT pathway involving both reversible steps and conformational changes (Mechanism C).

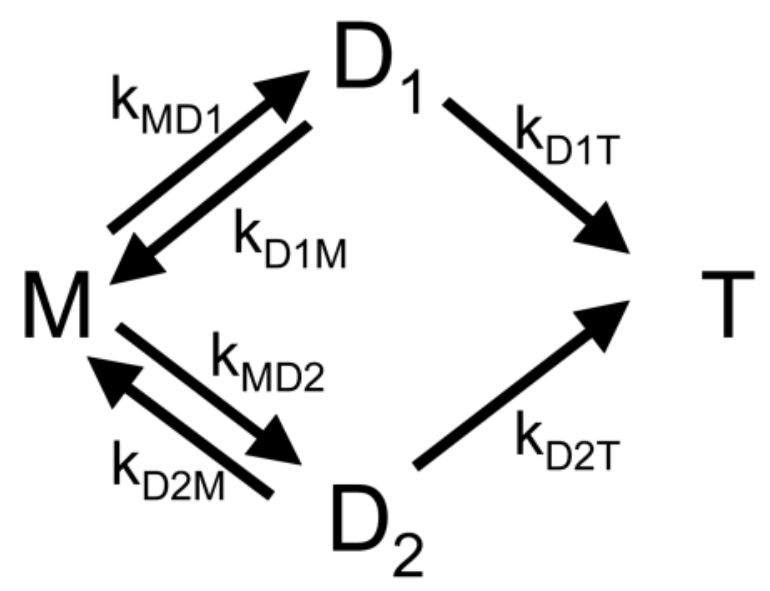

Mechanism D. 
The symmetry of TTR tetramer suggests that two distinct dimeric intermediates could be populated along the reaction pathway (Mechanism D). This two dimer mechanism will be tested employing reversible steps for both dimerization processes.

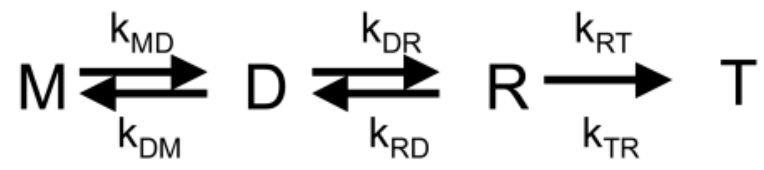

Mechanism E.

Finally, the MDRT mechanism will be scrutinized with reversible dimerization and trimerization processes in the reaction pathway (Mechanism E).

\section{Reassembly of TTR tetramers cannot occur by a simple series of irreversible bimolecular reactions - Denatured TTR does not reassemble by Mechanisms A} or $\mathbf{B}-$ Whether or not an assembly process is a series of irreversible bimolecular reactions can be determined by plotting the $\log \mathrm{t}_{50}$ (time required to reach $50 \%$ completion) versus the log of the TTR concentration. If the reassembly of TTR tetramer proceeds by a series of bimolecular steps (Mechanism A or B), the $\log \mathrm{t}_{50}$ versus $\log [\mathrm{TTR}]_{\text {monomer }}$ would exhibit a slope of -1 (40). Alternatively, a non-linear plot would indicate the presence of a first-order kinetic process in the reassembly pathway, which becomes increasingly important with increasing protein concentration. The plot of $\log \mathrm{t}_{50}$ versus $\log [\mathrm{TTR}]_{\text {monomer }}$ reveals a linear relationship over the concentration range of $0.72-4.32 \mu \mathrm{M}$ (monomer) with a slope of -1.4 (Figure 6). However, as the concentration increases above $4.32 \mu \mathrm{M}$, the plot deviates from linearity (Figure 6). This indicates the presence of a first-order kinetic process in the reassembly pathway. The first-order kinetic process associated with this deviation could be either a conformational change, or a dissociation step along the reaction pathway. These results demonstrate that TTR reassembly does not proceed by the irreversible mechanisms A and B.

\section{Global fitting analysis of the concentration dependent TTR reassembly time courses demonstrates that TTR does not reassemble by Mechanisms C or D-} In order to evaluate whether TTR reassembles by mechanisms $\mathrm{C}-\mathrm{E}$, the concentration dependent reassembly kinetics were compared to what would be predicted by the mathematical models of TTR reassembly by these mechanisms. Both monomer refolding (20) and ligand binding are known to be very fast processes (on the msec time scale; P. Hammarstrom, unpublished results); therefore, these steps were not included in the fitting analysis. The reassembly results were only fit to $80 \%$ completion, eliminating the potential influence of a tetramer dissociation process that could complicate the fitting analysis. Elimination of the final $20 \%$ of the reaction process also prevents the end-stage of the reaction from being too influential (it takes significantly longer to proceed from $80-100 \%$ completion than from 0 $80 \%$ completion, so the final $20 \%$ of the reaction tends to be over-represented in the data set despite its poor information content).

The simplest model of TTR reassembly that includes first-order kinetic processes is mechanism C. The rate equations associated with this mechanism are listed below.

$$
\begin{aligned}
& \frac{d[X]}{d t}=-2 k_{\mathrm{MD}^{\prime}}[X]^{2}+2 k_{D^{\prime} M}\left[X_{2}^{\prime}\right] \\
& \frac{d\left[X_{2}^{\prime}\right]}{d[t}=k_{\mathrm{MD}^{\prime}}[X]^{2}-k_{D^{\prime} M}\left[X_{2}^{\prime}\right]-k_{D^{\prime} D}\left[X_{2}^{\prime}\right]+k_{\mathrm{DD}^{\prime}}\left[X_{2}\right] \\
& \frac{d\left[X_{2}\right]}{d t}=k_{D^{\prime} D}\left[X_{2}^{\prime}\right]-k_{\mathrm{DD}^{\prime}}\left[X_{2}\right]-2 k_{\mathrm{DT}^{\prime}}\left[X_{2}\right]^{2}+2 k_{T^{\prime} D}\left[X_{4}^{\prime}\right] \\
& \frac{d\left[X_{4}^{\prime}\right]}{\mathrm{dt}}=k_{\mathrm{DT}^{\prime}}\left[X_{2}\right]^{2}-k_{T^{\prime} D}\left[X_{4}^{\prime}\right]-k_{T^{\prime} T}\left[X_{4}^{\prime}\right] \\
& \frac{d\left[X_{4}\right]}{\mathrm{dt}}=k_{T^{\prime} T}\left[X_{4}^{\prime}\right]
\end{aligned}
$$


The best global fits of the experimental data to Mechanism C do not accurately predict the TTR reassembly kinetics (Table 1A; Figure 7A). These results strongly suggest that TTR does not reassemble by the pathway described in Mechanism C.

Mechanism D was similarly fit to the concentration dependent TTR reassembly time course using the rate equations listed below.

$$
\begin{aligned}
& \frac{d[M]}{\mathrm{dt}}=-2 k_{\mathrm{MD}_{1}}[M]^{2}+2 k_{D_{1} M}\left[D_{1}\right]-2 k_{\mathrm{MD}_{2}}[M]^{2}+2 k_{D_{2} M}\left[D_{2}\right] \\
& \frac{d\left[D_{1}\right]}{\mathrm{dt}}=k_{\mathrm{MD}_{1}}[M]^{2}-k_{D_{1} M}\left[D_{1}\right]-2 k_{D_{1} T}\left[D_{1}\right]^{2} \\
& \frac{d\left[D_{2}\right]}{d t}=k_{\mathrm{MD}_{2}}[M]^{2}-k_{D_{2} M}\left[D_{2}\right]-2 k_{D_{2} T}\left[D_{2}\right]^{2} \\
& \frac{d[T]}{\mathrm{dt}}=k_{D_{1} T}\left[D_{1}\right]^{2}+k_{D_{2} T}\left[D_{2}\right]^{2}
\end{aligned}
$$

Global fitting of the experimental data to Mechanism D was improved in comparison to Mechanism C, but as the concentration of TTR increases, Mechanism D became less effective at predicting the TTR reassembly time courses (Table 1B; Figure 7B). These results strongly suggest that TTR homotetramers do not reassemble by Mechanism D.

$$
\begin{aligned}
& \text { Global fitting of the concentration dependent TTR reassembly time courses } \\
& \text { demonstrates that TTR reassembly is accurately predicted by Mechanism E- } \\
& \text { Mechanism E was fit to the concentration dependent TTR reassembly data using the rate } \\
& \text { equations listed below. } \\
& \frac{d[M]}{\mathrm{dt}}=-2 k_{\mathrm{MD}}[M]^{2}+2 k_{\mathrm{DM}}[D]-k_{\mathrm{DR}}[D][M]+k_{\mathrm{RD}}[R]-k_{\mathrm{RT}}[R][M] \\
& \frac{d[D]}{d \mathrm{dt}}=k_{\mathrm{MD}}[M]^{2}-k_{\mathrm{DM}}[D]-k_{\mathrm{DR}}[D][M]+k_{\mathrm{RD}}[R] \\
& \frac{d[R]}{\mathrm{dt}}=k_{\mathrm{DR}}[D][M]-k_{\mathrm{RD}}[R]-k_{\mathrm{RT}}[R][M] \\
& \frac{d[T]}{\mathrm{dt}}=k_{\mathrm{RT}}[R][M]
\end{aligned}
$$
demonstrates that TTR reassembly is accurately predicted by Mechanism EMechanism E was fit to the concentration dependent TTR reassembly data using the rate

The best fits of the experimental data to that predicted by a mathematical model of Mechanism E accurately predicts the TTR homotetramer reassembly time courses over a wide range of concentrations (Table 1C; Figure 7C). These results suggest that TTR homotetramers reassemble by the sequential addition of monomers to dimeric and trimeric assembly intermediates. Fitting the concentration dependent reassembly kinetics to more complicated variations of Mechanism E (e.g. incorporating first order reorganization steps or allowing the existence of a second type of dimeric species) can slightly increase the accuracy of the fit (Supporting Information), but this modest increase in accuracy does not merit increasing the number of parameters in the model. The existence of first-order reorganization steps cannot be rigorously excluded based on our data, but the above results show that Mechanism E is the simplest, best-fitting mechanism for TTR tetramer reassembly from monomers.

\section{Discussion}

\section{TTR tetramers reassemble by an energetically balanced MDRT mechanism}

Herein, we have demonstrated that the reassembly of TTR is best described by Mechanism E, in which TTR monomers are sequentially added to dimeric and trimeric intermediates, with both dimer formation and trimer formation being reversible processes (although fast conformational rearrangements could not be rigorously excluded with our current data set). The rate constants emerging from the best fits to the experimental data reveal that TTR reassembly is kinetically balanced (Table 1C). The initial bimolecular reaction between two TTR monomers, forming a dimeric intermediate, is found to be unfavorable at low $\mu \mathrm{M}$ concentrations. The fit is insensitive to the absolute value of the dimerization rate constants, but the thermodynamic equilibrium constant $\left(\mathrm{K}_{\mathrm{dimer}}\right)$ defined by the ratio of the dissociation 
and association constants $\left(\mathrm{K}_{\mathrm{dimer}}=\mathrm{k}_{\mathrm{DM}} / \mathrm{k}_{\mathrm{MD}}=28 \mu \mathrm{M}\right.$; Table $\left.1 \mathrm{C}\right)$ is critical. This suggests that dimerization reaches a rapid pre-equilibrium, which is achieved on a timescale that is fast relative to the subsequent steps along the reassembly pathway. Trimer formation is also found to be a reversible process, which is more favorable than dimerization $\left(\right.$ Table $1 \mathrm{C} ; \mathrm{K}_{\text {trimer }}=$ $\mathrm{k}_{\mathrm{RD}} / \mathrm{k}_{\mathrm{DR}}=7.5 \mathrm{nM}$ ). The rate constants for trimer formation (Table $1 \mathrm{C} ; \mathrm{k}_{\mathrm{DR}}=7.2 \times 10^{5} \mathrm{M}^{-1}$ $\mathrm{s}^{-1}$ ) and trimer dissociation (Table $1 \mathrm{C} ; \mathrm{k}_{\mathrm{RD}}=5.7 \times 10^{-3} \mathrm{~s}^{-1}$ ) were found to be well-defined by the fitting analysis, demonstrating that trimer formation is the commitment step along the reassembly pathway, effectively pulling protein through the unfavorable monomer-dimer equilibrium. The rate of tetramer formation by addition of monomer to the trimeric intermediate is found to be the slowest step along the forward reassembly pathway (Table $1 \mathrm{C} ; \mathrm{k}_{\mathrm{RT}}=$ $7.8 \times 10^{4} \mathrm{M}^{-1} \mathrm{~s}^{-1}$ ), a full order of magnitude slower than trimer formation. Since tetramer formation is dependent on the concentration of monomer, the fate of the trimeric intermediate is influenced by the extent of reassembly reaction. At early time points along the reassembly pathway, tetramer formation is favored because the relatively high monomer concentration increases the rate of the forward tetramer forming step (Figure 8A). As the concentration of monomer becomes depleted, the rate of tetramer formation decreases, increasing the amount of trimer undergoing dissociation to the unstable dimeric intermediate, slowing the overall assembly of TTR homotetramers (Figure 8A).

Previously, it was reported that the MDT mechanism of homotetramer reassembly would be evolutionarily favored over an MDRT mechanism, which is consistent with the high frequency by which the MDT mechanism applies (41). The MDRT pathway was believed to be disadvantageous because with anything but optimal rate constants, monomeric protein could be rapidly depleted to form assembly intermediates. The low concentration of monomer in solution following its rapid depletion would effectively slow the forward steps of the MDRT pathway (monomer addition to either a dimer or trimer), resulting in the buildup of potentially harmful reassembly intermediates. TTR avoids this potential pitfall with its MDRT mechanism by incorporating reversible dimerization and trimerization steps into the pathway. TTR reassembles by rapid monomer depletion, resulting in a buildup of the trimer concentration, which can either proceed to tetramer by monomer addition or dissociate to dimer and subsequently to monomer. Low monomer concentrations decrease the rate of tetramer formation and increase the amount of trimer undergoing dissociation, populating the unstable dimeric intermediate, which rapidly dissociates to monomers. In other words the kinetic balance between the reversible dimer-forming and trimer-forming steps allows the reassembly of TTR tetramers to proceed to completion without the irreversible population of potentially harmful reassembly intermediates.

\section{Tetramer reassembly during subunit exchange under native conditions occurs by a different mechanism than tetramer reassembly from denatured monomers}

TTR tetramers are known to exchange subunits under native conditions $(38,39)$. We have recently shown that this process occurs by a rate limiting dissociation of the tetramer to two dimeric intermediates (42). These dimers rapidly dissociate to monomers, and then reassemble to form tetramers. According to the principle of microscopic reversibility, the reassembly to tetramers must occur by the reverse of the mechanism outlined above. Thus, tetramer reassembly during subunit exchange under native conditions occurs by an MDT mechanism. In contrast, as stated above, the results described herein indicate that TTR reassembly starting from a pool of denatured monomers occurs by an MDRT mechanism. These observations illustrate how protein reassembly mechanisms can depend on conditions. The high initial concentration of TTR monomers that exists when TTR is reconstituted from denatured monomers forces the protein to reassemble by the energetically balanced MDRT mechanism described above. However, the concentration of monomers under native conditions is very low, so TTR reassembles by an MDT mechanism during subunit exchange, probably because the 
rate of tetramer reassembly by an MDT mechanism does not explicitly depend on monomer concentration at each step (see the rate equations for Mechanisms A, C, and D), unlike the rate of tetramer reassembly by an MDRT mechanism (see the rate equations for Mechanisms B and E).

\section{TTR monomers are kinetically partitioned between the aggregation and reassembly pathways}

TTR monomers resulting from cooperative homotetramer dissociation can be sequestered into one of two pathways, aggregation or reassembly (Figure 8C). The partitioning of protein into these two pathways depends on three parameters: 1 ) The relative concentrations of natively folded monomer and the amyloidogenic intermediate, 2) the relative rate constants of the reassembly and aggregation process, and 3 ) the relative concentrations of reassembly intermediates and soluble TTR aggregates. Factors that influence any of these parameters will affect the partitioning of TTR between these two pathways, potentially providing a mechanistic model to understand the late age of onset of TTR-related amyloid diseases.

Aggregation and reassembly are separated by a folding/misfolding equilibrium defining the relative concentrations of the natively folded monomer and the aggregation competent amyloidogenic intermediate. Misfolding of the TTR monomer is unfavorable under physiological conditions, but environments that enable misfolding could dramatically influence the steady-state distribution of folded and misfolded monomers in solution. This perturbation of the steady-state concentrations of monomeric conformations would increase the population of misfolded monomer, effectively increasing the total population of TTR entering into the aggregation pathway. Similarly, mutations in the TTR amino acid sequence can also affect the steady-state populations of folded and misfolded TTR monomers. Mutations that destabilize the monomer fold would increase the concentration of the aggregation competent intermediate and the amount of protein partitioning into the aggregation pathway, potentially influencing the disease onset and pathology.

Partitioning of TTR between the reassembly and aggregation pathways is also influenced by the relative kinetics of the two processes. As demonstrated by the reassembly fitting analysis, the dimerization step is unfavorable at low monomer concentrations $(<1 \mu \mathrm{M})$ and rapidly affords a pre-equilibrium between monomer and dimer favoring the monomer. Therefore, the trimer formation reaction is the first important kinetic step along the reassembly reaction pathway, with a rate not only dependent on the rate constant and the concentration of folded monomer in solution, but also on the concentration of the dimeric intermediate. In contrast, the competing aggregation rate of misfolded TTR monomers is dependent on both the rate constant of aggregation, the concentration of misfolded monomer, and the total concentration of soluble aggregates in solution.

Influencing the rate constants for either aggregation or reassembly can affect the steady-state partitioning of TTR between these competing pathways. Although the rate constants associated with the aggregation pathway are unknown, it is likely that these would be affected by environmental factors. In addition, point mutations in the TTR sequence that alter rate constants associated with reassembly, perhaps by destabilizing dimeric or trimeric intermediates, would decrease the amount of protein entering into the reassembly pathway and increase the amount of protein entering into the aggregation pathway. Destabilization of reassembly intermediates would result in a redistribution of protein from the reassembly pathway to the aggregation pathway, potentially explaining the earlier age of onset of familial TTR amyloid disease.

The partitioning of TTR between the aggregation and reassembly pathways is also influenced by the concentrations of soluble TTR aggregates and reassembly intermediates in solution. Under conditions where the concentration of natively folded TTR oligomers (monomer, dimer, 
and trimer) are higher than that of soluble TTR aggregates, TTR will kinetically partition more effectively into the reassembly pathway. As the concentration of soluble aggregates increases the rate of aggregation similarly increases. This is a consequence of the downhill polymerization mechanism governing TTR aggregation, wherein a bimolecular collision between two misfolded monomers has a rate constant identical to the bimolecular reaction between a misfolded monomer and a soluble aggregate. Therefore, increases in the concentration of soluble TTR aggregates relative to the concentration of tetramer reassembly intermediates would allow protein to partition more effectively into the aggregation pathway.

Since aggregation becomes more favorable as the concentration of soluble aggregates increases, the kinetic partitioning of TTR into the reassembly and aggregation pathway may be time-dependent. If the in vivo clearance of soluble TTR aggregates becomes slower than the synthesis of TTR aggregates, then the concentration of soluble aggregates in the extracellular space will increase over time, increasing the proportion of TTR partitioning into the aggregation pathway. This would suggest that TTR is constantly being kinetically partitioned between aggregation and reassembly, and as the concentration of soluble aggregates in the serum increases, the aggregation pathway becomes more efficient, resulting in pathology. This time dependent change in partitioning into the aggregation pathway may partially explain and influence the late age onset of TTR-related amyloid diseases. Unfortunately, the relative rates and mechanisms of clearance of soluble TTR aggregates and TTR tetramers are unknown, nonetheless this framework may prove important for understanding the late age of onset for TTR amyloid diseases.

\section{Conclusions}

Herein, we demonstrate that wild-type TTR reassembles by an MDRT pathway, where TTR tetramers are formed by the sequential addition of monomers to reassembly intermediates. Reassembly competes effectively with the TTR aggregation pathway for TTR monomers in the extracellular space until soluble TTR aggregates start to accumulate, favoring partitioning towards the aggregation pathway, likely influencing the pathology of TTR related amyloid diseases. These results suggest that protein may be partitioned between these two competing pathways in a time-dependent process, because aggregation becomes more favorable as the concentration of soluble aggregates increases possibly resulting from an age-related slowing in the clearance of soluble aggregates or changing solution conditions that would increase the amyloidogenic intermediate concentration relative to folded monomer concentration. This kinetic partitioning mechanism may be an important factor in the pathogenesis of TTR related amyloid diseases. A better understanding of the mechanism of TTR tetramer and TTR soluble aggregate clearance from the serum as a function of age should enhance our ability to therapeutically intervene in these diseases because we would know when to begin prophylactic strategies such as small-molecule mediated kinetic stabilization of the non-amyloidogenic native state (35).

\section{Supplementary Material}

Refer to Web version on PubMed Central for supplementary material.

\section{Acknowledgements}

We would like to thank Dr. Patrick Braun, Ted Foss, and Dr. Per Hammarström for helpful discussions.

\section{Abbreviations}

TTR

Transthyretin 
SSA

Senile Systemic Amyloidosis

FAP

Familial Amyloid Polyneuropathy

FAC

Familial Amyloid Cardiomyopathy

CNSA

Central nervous system amyloidosis

MDT

Monomer-dimer-tetramer

MDRT

Monomer- dimer- trimer- tetramer

ITC

Isothermal titration calorimetry

\section{References}

1. Buxbaum JN. The systemic amyloidoses. Curr Opin Rheumatol 2004;16:67-75. [PubMed: 14673392]

2. Petkova AT, Leapman RD, Guo Z, Yau WM, Mattson MP, Tycko R. Self-propagating, molecularlevel polymorphism in Alzheimer's beta-amyloid fibrils. Science 2005;307:262-265. [PubMed: 15653506]

3. Selkoe DJ. Cell biology of protein misfolding: the examples of Alzheimer's and Parkinson's diseases. Nat Cell Biol 2004;6:1054-1061. [PubMed: 15516999]

4. Colon W, Kelly JW. Partial denaturation of transthyretin is sufficient for amyloid fibril formation in vitro. Biochemistry 1992;31:8654-8660. [PubMed: 1390650]

5. Speed MA, Wang DI, King J. Specific aggregation of partially folded polypeptide chains: the molecular basis of inclusion body composition. Nat Biotechnol 1996;14:1283-1287. [PubMed: 9631094]

6. Chiti F, Taddei N, Baroni F, Capanni C, Stefani M, Ramponi G, Dobson CM. Kinetic partitioning of protein folding and aggregation. Nat Struct Biol 2002;9:137-143. [PubMed: 11799398]

7. Hamilton JA, Benson MD. Transthyretin: a review from a structural perspective. Cell Mol Life Sci 2001;58:1491-1521. [PubMed: 11693529]

8. Liu K, Cho HS, Lashuel HA, Kelly JW, Wemmer DE. A glimpse of a possible amyloidogenic intermediate of transthyretin. Nat Struct Biol 2000;7:754-757. [PubMed: 10966644]

9. Sekijima Y, Hammarstrom P, Matsumura M, Shimizu Y, Iwata M, Tokuda T, Ikeda S, Kelly JW. Energetic characteristics of the new transthyretin variant A25T may explain its atypical central nervous system pathology. Lab Invest 2003;83:409-417. [PubMed: 12649341]

10. Sekijima Y, Wiseman RL, Matteson J, Hammarstrom P, Miller SR, Sawkar AR, Balch WE, Kelly JW. The Biological and Chemical Basis for Tissue Selective Amyloid Disease. Cell 2005;121:7385. [PubMed: 15820680]

11. Hammarstrom P, Sekijima Y, White JT, Wiseman RL, Lim A, Costello CE, Altland K, Garzuly F, Budka H, Kelly JW. D18G transthyretin is monomeric, aggregation prone, and not detectable in plasma and cerebrospinal fluid: a prescription for central nervous system amyloidosis? Biochemistry 2003;42:6656-6663. [PubMed: 12779320]

12. Reixach N, Deechongkit S, Jiang X, Kelly JW, Buxbaum JN. Tissue damage in the amyloidoses: Transthyretin monomers and nonnative oligomers are the major cytotoxic species in tissue culture. Proc Natl Acad Sci U S A 2004;101:2817-2822. [PubMed: 14981241]

13. Buxbaum JN, Tagoe CE. The genetics of the amyloidoses. Annu Rev Med 2000;51:543-569. [PubMed: 10774481] 
14. Westermark P, Sletten K, Johansson B, Cornwell GG 3rd. Fibril in senile systemic amyloidosis is derived from normal transthyretin. Proc Natl Acad Sci U S A 1990;87:2843-2845. [PubMed: 2320592]

15. Jiang X, Buxbaum JN, Kelly JW. The V122I cardiomyopathy variant of transthyretin increases the velocity of rate-limiting tetramer dissociation, resulting in accelerated amyloidosis. Proc Natl Acad Sci U S A 2001;98:14943-14948. [PubMed: 11752443]

16. McCutchen SL, Lai Z, Miroy GJ, Kelly JW, Colon W. Comparison of lethal and nonlethal transthyretin variants and their relationship to amyloid disease. Biochemistry 1995;34:13527-13536. [PubMed: 7577941]

17. Jacobson DR, Pastore RD, Yaghoubian R, Kane I, Gallo G, Buck FS, Buxbaum JN. Variant-sequence transthyretin (isoleucine 122) in late-onset cardiac amyloidosis in black Americans. N Engl J Med 1997;336:466-473. [PubMed: 9017939]

18. Sousa A, Coelho T, Barros J, Sequeiros J. Genetic epidemiology of familial amyloidotic polyneuropathy (FAP)-type I in Povoa do Varzim and Vila do Conde (north of Portugal). Am J Med Genet 1995;60:512-521. [PubMed: 8825887]

19. Ikeda S, Nakazato M, Ando Y, Sobue G. Familial transthyretin-type amyloid polyneuropathy in Japan: clinical and genetic heterogeneity. Neurology 2002;58:1001-1007. [PubMed: 11940682]

20. Jiang X, Smith CS, Petrassi HM, Hammarstrom P, White JT, Sacchettini JC, Kelly JW. An engineered transthyretin monomer that is nonamyloidogenic, unless it is partially denatured. Biochemistry 2001;40:11442-11452. [PubMed: 11560492]

21. Lai Z, Colon W, Kelly JW. The acid-mediated denaturation pathway of transthyretin yields a conformational intermediate that can self-assemble into amyloid. Biochemistry 1996;35:6470-6482. [PubMed: 8639594]

22. Lashuel HA, Lai Z, Kelly JW. Characterization of the transthyretin acid denaturation pathways by analytical ultracentrifugation: implications for wild-type, V30M, and L55P amyloid fibril formation. Biochemistry 1998;37:17851-17864. [PubMed: 9922152]

23. Hurshman AR, White JT, Powers ET, Kelly JW. Transthyretin aggregation under partially denaturing conditions is a downhill polymerization. Biochemistry 2004;43:7365-7381. [PubMed: 15182180]

24. Hammarstrom P, Jiang X, Hurshman AR, Powers ET, Kelly JW. Sequence-dependent denaturation energetics: A major determinant in amyloid disease diversity. Proc Natl Acad Sci U S A 2002;99:16427-16432. [PubMed: 12351683]

25. Girg R, Rudolph R, Jaenicke R. The dimeric intermediate on the pathway of reconstitution of lactate dehydrogenase is enzymatically active. FEBS Lett 1983;163:132-135. [PubMed: 6628683]

26. Bernhardt G, Rudolph R, Jaenicke R. Reassociation of lactic dehydrogenase from pig heart studied by cross-linking with glutaraldehyde. Z Naturforsch [C] 1981;36:772-777.

27. Hermann R, Jaenicke R, Rudolph R. Analysis of the reconstitution of oligomeric enzymes by crosslinking with glutaraldehyde: kinetics of reassociation of lactic dehydrogenase. Biochemistry 1981;20:5195-5201. [PubMed: 6794607]

28. Hermann R, Rudolph R, Jaenicke R, Price NC, Scobbie A. The reconstitution of denatured phosphoglycerate mutase. J Biol Chem 1983;258:11014-11019. [PubMed: 6309828]

29. Hermann R, Jaenicke R, Price NC. Evidence for active intermediates during the reconstitution of yeast phosphoglycerate mutase. Biochemistry 1985;24:1817-1821. [PubMed: 2990542]

30. Nichtl A, Buchner J, Jaenicke R, Rudolph R, Scheibel T. Folding and association of betaGalactosidase. J Mol Biol 1998;282:1083-1091. [PubMed: 9753555]

31. Rehaber V, Jaenicke R. Stability and reconstitution of D-glyceraldehyde-3-phosphate dehydrogenase from the hyperthermophilic eubacterium Thermotoga maritima. J Biol Chem 1992;267:10999_ 11006. [PubMed: 1366231]

32. Mateu MG, Sanchez Del Pino MM, Fersht AR. Mechanism of folding and assembly of a small tetrameric protein domain from tumor suppressor p53. Nat Struct Biol 1999;6:191-198. [PubMed: 10048932]

33. Risse B, Stempfer G, Rudolph R, Mollering H, Jaenicke R. Stability and reconstitution of pyruvate oxidase from Lactobacillus plantarum: dissection of the stabilizing effects of coenzyme binding and subunit interaction. Protein Sci 1992;1:1699-1709. [PubMed: 1304899] 
34. Lashuel HA, Wurth C, Woo L, Kelly JW. The most pathogenic transthyretin variant, L55P, forms amyloid fibrils under acidic conditions and protofilaments under physiological conditions. Biochemistry 1999;38:13560-13573. [PubMed: 10521263]

35. Hammarstrom P, Wiseman RL, Powers ET, Kelly JW. Prevention of transthyretin amyloid disease by changing protein misfolding energetics. Science 2003;299:713-716. [PubMed: 12560553]

36. Hammarstrom P, Schneider F, Kelly JW. Trans-suppression of misfolding in an amyloid disease. Science 2001;293:2459-2462. [PubMed: 11577236]

37. Green NS, Palaninathan SK, Sacchettini JC, Kelly JW. Synthesis and characterization of potent bivalent amyloidosis inhibitors that bind prior to transthyretin tetramerization. J Am Chem Soc 2003;125:13404-13414. [PubMed: 14583036]

38. Schneider F, Hammarstrom P, Kelly JW. Transthyretin slowly exchanges subunits under physiological conditions: A convenient chromatographic method to study subunit exchange in oligomeric proteins. Protein Sci 2001;10:1606-1613. [PubMed: 11468357]

39. Wiseman RL, Green NS, Kelly JW. Kinetic Stabilization of an Oligomeric Protein under Physiological Conditions Demonstrated by a Lack of Subunit Exchange: Implications for Transthyretin Amyloidosis. Biochemistry. 2005in press

40. Goldstein RF, Stryer L. Cooperative polymerization reactions. Analytical approximations, numerical examples, and experimental strategy. Biophys J 1986;50:583-599. [PubMed: 3779001]

41. Powers ET, Powers DL. A perspective on mechanisms of protein tetramer formation. Biophys J 2003;85:3587-3599. [PubMed: 14645052]

42. Foss T, Wiseman RL, Kelly JW. The Pathway by Which the Tetrameric Protein Transthyretin Dissociates. Biochemistry. 2005In press

43. Foss TR, Kelker MS, Wiseman RL, Wilson IA, Kelly JW. Kinetic stabilization of the native state by protein engineering: implications for inhibition of transthyretin amyloidogenesis. J Mol Biol 2005;347:841-854. [PubMed: 15769474] 
A

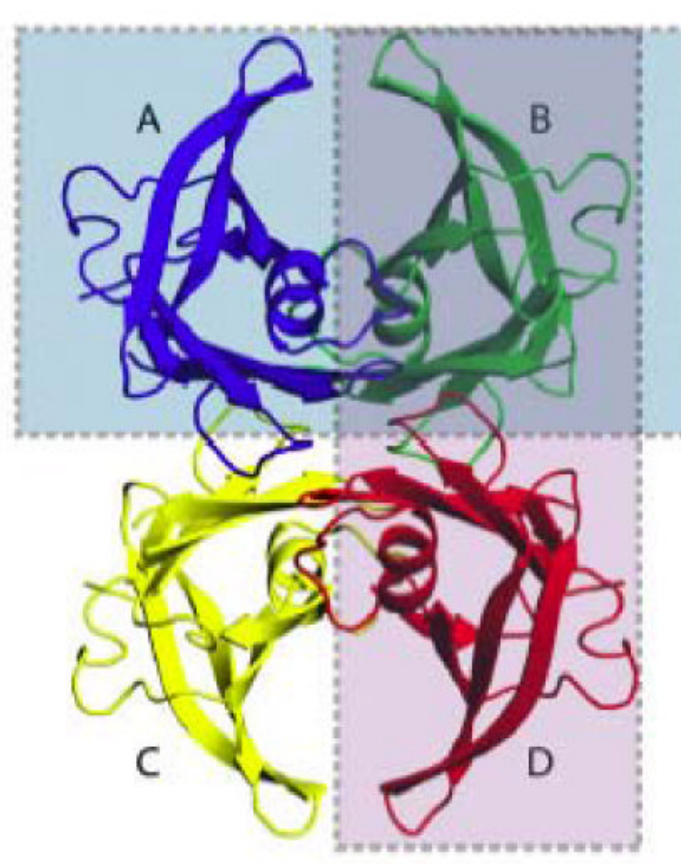

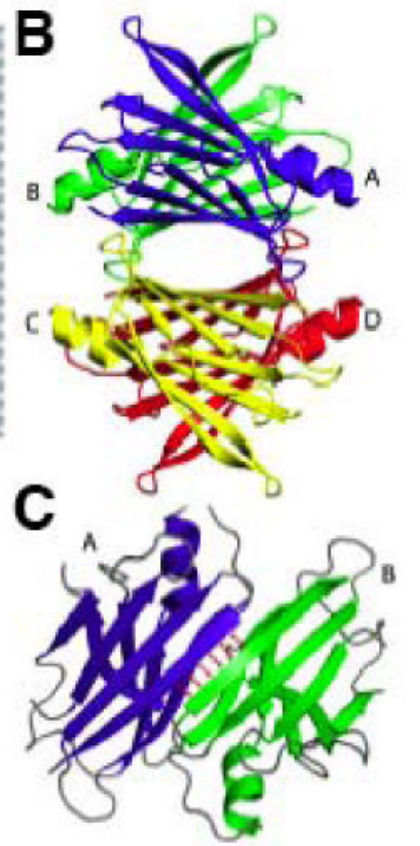

Figure 1.

Ribbon diagram depiction of the crystal structure of the TTR tetramer demonstrating the two distinct dimeric interfaces that comprise the tetrameric structure. A) Ribbon diagram of the entire TTR tetramer highlighting the two distinct dimeric interfaces (purple and green boxes). B) A $90^{\circ}$ rotation of the image in Figure $1 \mathrm{~A}$ indicating the dimeric interface that composes the thyroxine binding site of the TTR tetramer (purple box in Figure 1A; comprised of the green and red TTR subunits). C) Crystal structure of the second TTR dimeric interface (green box in Figure 1A; composed of the blue and green subunits). Figure adapted from (43). 


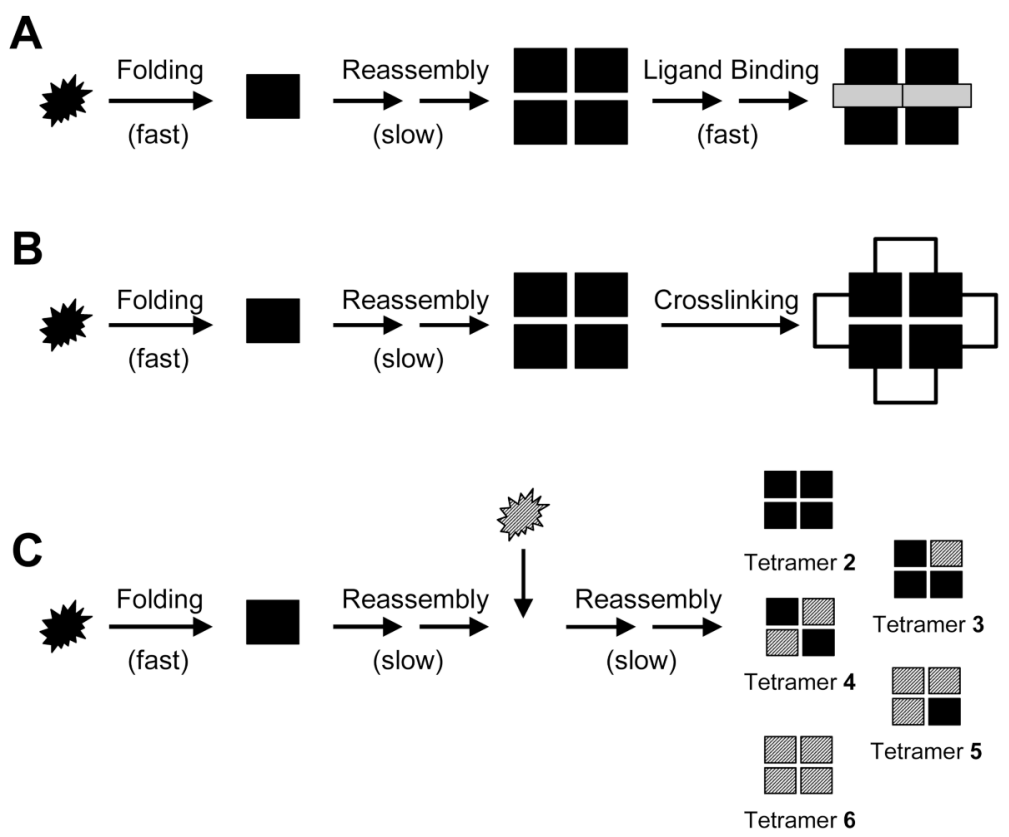

Figure 2.

Experimental methods used to measure the TTR reassembly time courses. A) Small molecule ligand binding fluorescence assay used to follow TTR tetramer reassembly. Denatured TTR is rapidly diluted into refolding buffer with a 10-fold molar excess of small molecule $\mathbf{1}$ (Gray bars). TTR tetramers are detected by small molecule binding ( $\mathbf{1}$ fluoresces only when bound to the TTR tetramer). B) Glutaraldehyde crosslinking analysis of TTR reassembly. TTR is diluted 1:10 into refolding buffer. At several time points, an aliquot of the reassembly reaction is removed and crosslinked by glutaraldehyde (black brackets). The amount of tetramer is determined by SDS-PAGE analysis. C) Subunit incorporation assay to measure the rate of TTR homotetramer reassembly. Denatured wild-type TTR (black symbols) was diluted 1:10 into refolding buffer and allowed to reassemble for variable periods of time. At the indicated time points, denatured flag-tag wild-type TTR (hatched symbols) was then diluted 1:10 into refolding buffer and added to the wild-type TTR reassembly reaction. Reassembly was then allowed to proceed to completion, resulting in the formation of tetramers 2-6 with varying stoichiometries of wild-type (black) and flag-tag wild-type (hatched) TTR subunits depending on the extent of wild type TTR homotetramer formation. The distributions of tetramers 2-6 were quantified by anion exchange chromatography. 
A<smiles>O=C(O)c1ccccc1Nc1cc(Cl)cc(Cl)c1</smiles>

\section{1 \\ $\mathrm{K}_{1}=2 \times 10^{8} \mathrm{M}^{-1}$ \\ $\mathrm{K}_{2}=2 \times 10^{8} \mathrm{M}^{-1}$}

B

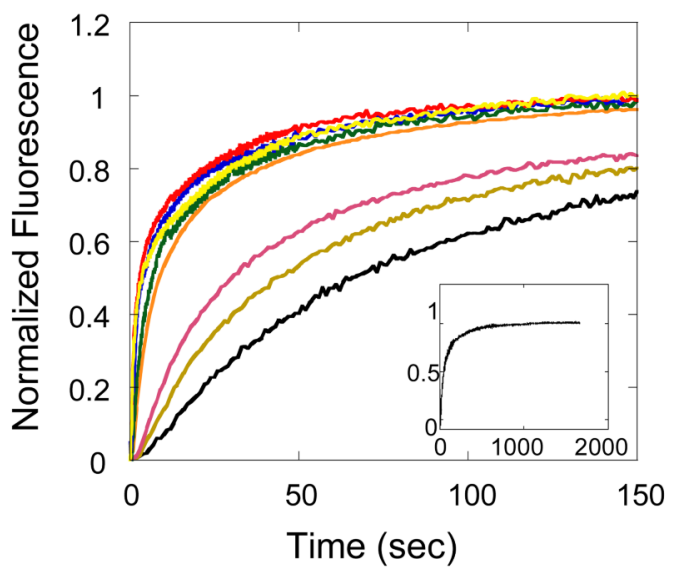

Figure 3.

TTR tetramer reassembly can be measured over a wide concentration range using the environment-sensitive fluorescence exhibited by 1 upon binding to TTR. A) Structure of small molecule 1 with binding constants, as determined by isothermal titration calorimetry. B) Concentration dependent reassembly time courses of wild type TTR homotetramers over a range of protein concentration $(0.72-36 \mu \mathrm{M}$; monomer) monitored by the binding and fluorescence of 1. $0.72 \mu \mathrm{M}$ - black, $1.08 \mu \mathrm{M}$ - brown, $1.44 \mu \mathrm{M}$ - pink, $4.32 \mu \mathrm{M}$ - orange, 7.2 $\mu \mathrm{M}$ - green, $14.4 \mu \mathrm{M}$ - blue, $21.6 \mu \mathrm{M}$ - red, $36 \mu \mathrm{M}$ - yellow. The inset reveals the complete reassembly time course of $1.08 \mu \mathrm{M}$ TTR (monomer). 
A

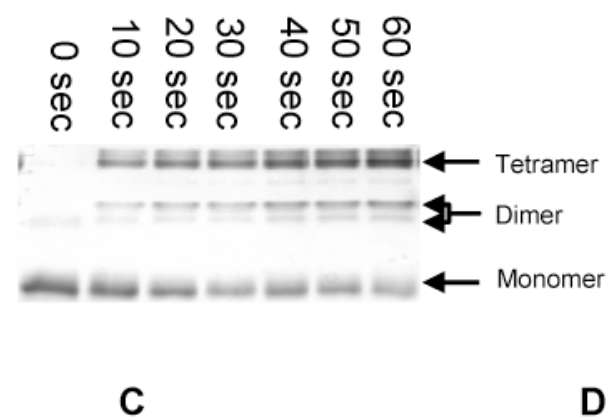

B
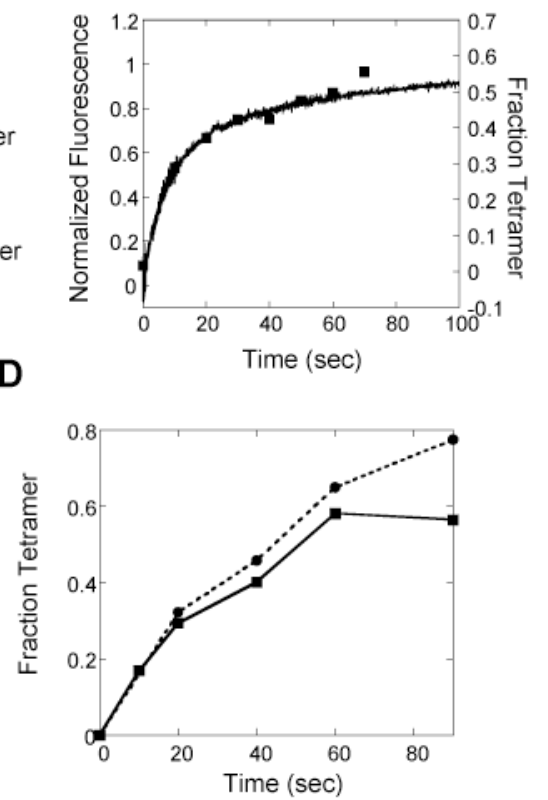

Figure 4.

Glutaraldehyde crosslinking analysis of TTR homotetramer reassembly. A) SDS-PAGE assessment of glutaraldehyde crosslinked oligomers that exist at various time points in TTR reassembly reactions. The reassembly reaction was allowed to proceed for the indicated time prior to crosslinking of the sample. The arrows indicate the different oligomeric species observed by SDS-PAGE analysis. B) Overlay of TTR reassembly ( $4.32 \mu \mathrm{M}$; monomer) as measured by the fluorescence of $\mathbf{1}$ (black line) and glutaraldehyde crosslinking (black squares). The two time courses are nearly identical indicating that both assays are measuring the same process. C) Glutaraldehyde crosslinking analysis of TTR reassembly $(4.32 \mu \mathrm{M}$; monomer) in the presence (circles and dashed line) or absence (squares and solid line) of small molecule 1. The nearly identical time courses demonstrate that the small molecule does not seem to affect the reassembly kinetics. D) Glutaraldehyde crosslinking analysis of TTR reassembly (1.44 $\mu \mathrm{M}$; monomer) in the presence (circles and dashed line) or absence (squares and solid line) of small molecule 1 . The nearly identical kinetics demonstrates that the small molecule does not appear to affect the reassembly kinetics. 
A
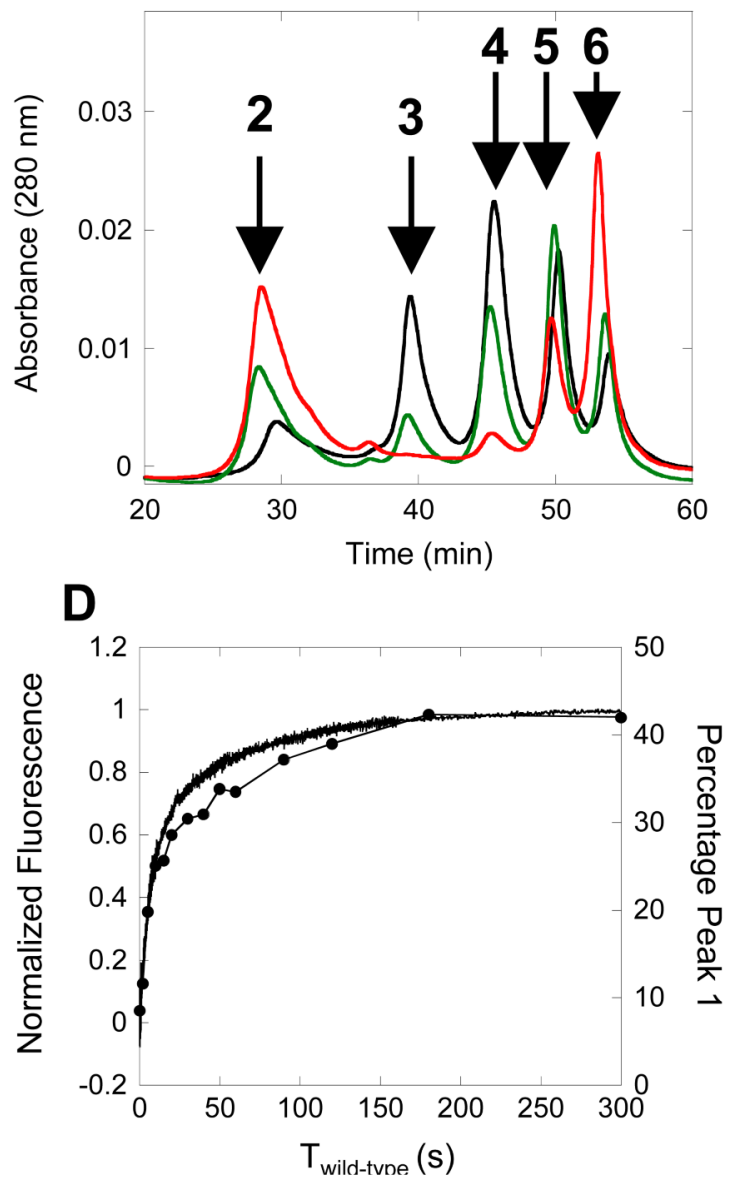

B

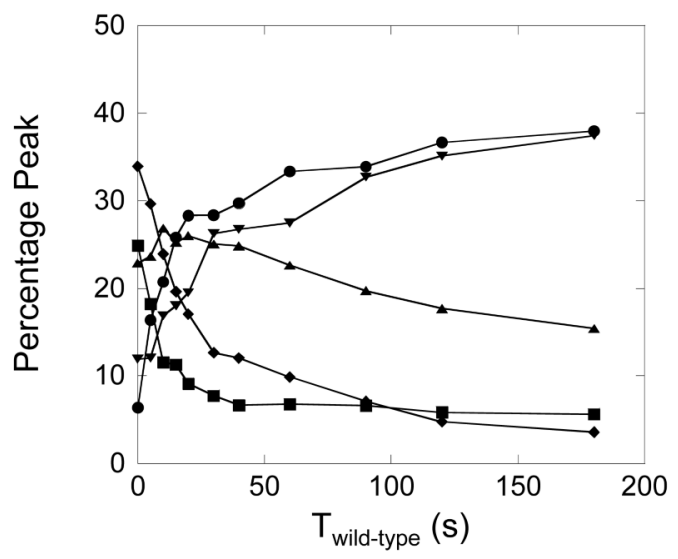

C

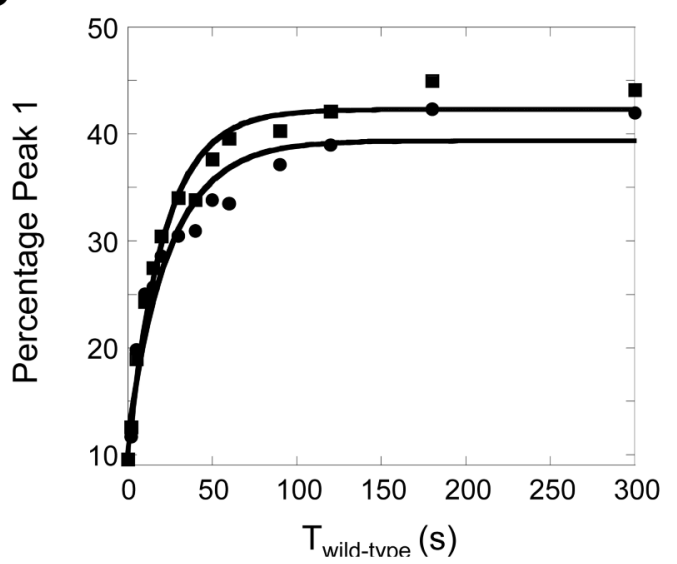

Figure 5.

TTR homotetramer reassembly can be monitored by a subunit incorporation assay. A) Anion exchange chromatogram of tetramers 2-6 after allowing the wild-type TTR $(4.32 \mu \mathrm{M}$; monomer) to reassemble in the absence of flag-tag wild-type TTR for varying times ( $0 \mathrm{sec}-$ black; $10 \mathrm{sec}$ - green; $180 \mathrm{sec}$ - red). Tetramers 2-6 (arrows) have different wild-type and flagtag wild-type TTR subunit stoichiometries (tetramer $\mathbf{2}$ - (wild-type TTR) 4 ; tetramer $\mathbf{3}$ (wild-

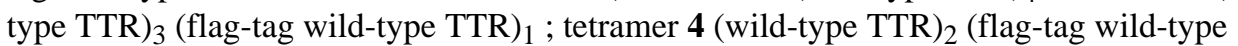
TTR) 2 ; tetramer 5 (wild-type TTR) 1 (flag-tag wild-type TTR) 3 ; tetramer $\mathbf{6}$ (flag-tag wild-type TTR $)_{4}$. B) Graph of the time dependent distribution of tetramers 2-6 as a function of the incubation time of wild-type TTR reassembly prior to the addition of flag-tag wild-type TTR $\left(\mathrm{t}_{\text {wild-type }}\right)$. Tetramer $\mathbf{2}$ - black circles; Tetramer $\mathbf{3}$-red squares; Tetramer $\mathbf{4}$-green diamonds ; Tetramer $\mathbf{5}$ - blue triangles ; Tetramer $\mathbf{6}$ - orange upside down triangles. C) Overlay of TTR homotetramer reassembly time courses $(4.32 \mu \mathrm{M}$; monomer) as measured by the fluorescence of 1 (black line) and by the subunit incorporation method (black circles). The nearly identical kinetics strongly suggests that subunit incorporation measures the same kinetic process as the ligand binding fluorescence assay. D) Graph of TTR tetramer reassembly (4.32 $\mu \mathrm{M}$; monomer) as measured by subunit incorporation in the presence (red squares) or absence (black circles) of small molecule $\mathbf{1}$. The similar time courses demonstrate that the small molecule does not interfere with the reassembly kinetics of TTR homotetramers. 


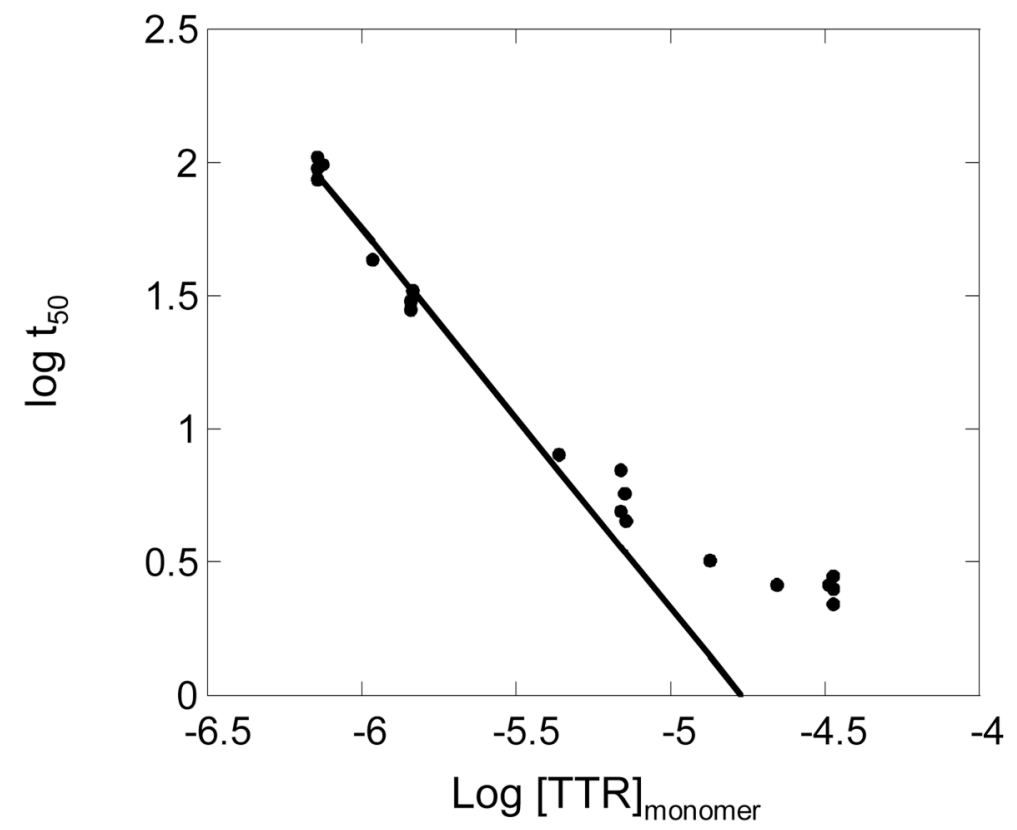

Figure 6.

Plot of the $\log \mathrm{t}_{50}$ versus the $\log \left([\mathrm{TTR}]_{\text {monomer }}\right)$ for the process of TTR tetramer reassembly. Protein concentrations below $4.32 \mu \mathrm{M}$ exhibit a linear relationship with a slope of -1.4. As the concentration of protein is increased to $>4.32 \mu \mathrm{M}$, the plot deviates from linearity indicating the presence of a first-order kinetic process in the reassembly pathway. 
A
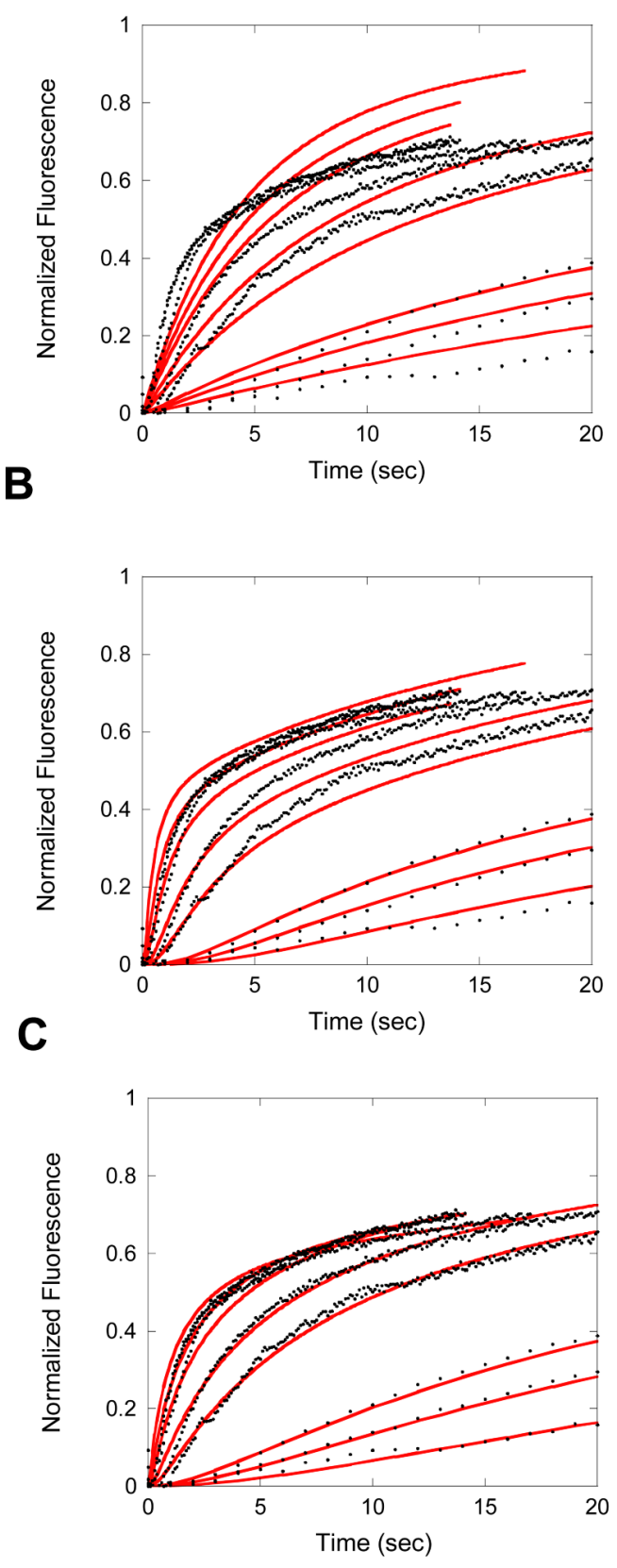

Figure 7.

Global fitting analysis of the concentration dependent TTR reassembly time courses. A) Global fitting analysis of the TTR reassembly kinetics to Mechanism C. The fits (red lines) do not accurately predict the kinetics of TTR homotetramer reassembly (black circles). B) Global fitting analysis of the TTR reassembly kinetics to Mechanism D. At low protein concentrations, the mechanistic fitting analysis (red lines) accurately predict the reassembly time courses (black symbols), but as the concentration of protein increases, the predicted fits are found to be poor, demonstrating that TTR homotetramers do not reassemble by Mechanism D. C) Global fitting analysis of TTR reassembly kinetics to Mechanism E. Mechanism E accurately predicts the reassembly time courses over the entire range of protein concentrations indicating that TTR 
tetramers reassemble by this mechanistic pathway. Fitting the concentration dependent reassembly kinetics to more complicated variations of Mechanism E (e.g. incorporating first order reorganization steps or allowing the existence of a second type of dimeric species) does not substantially improve the fit. 


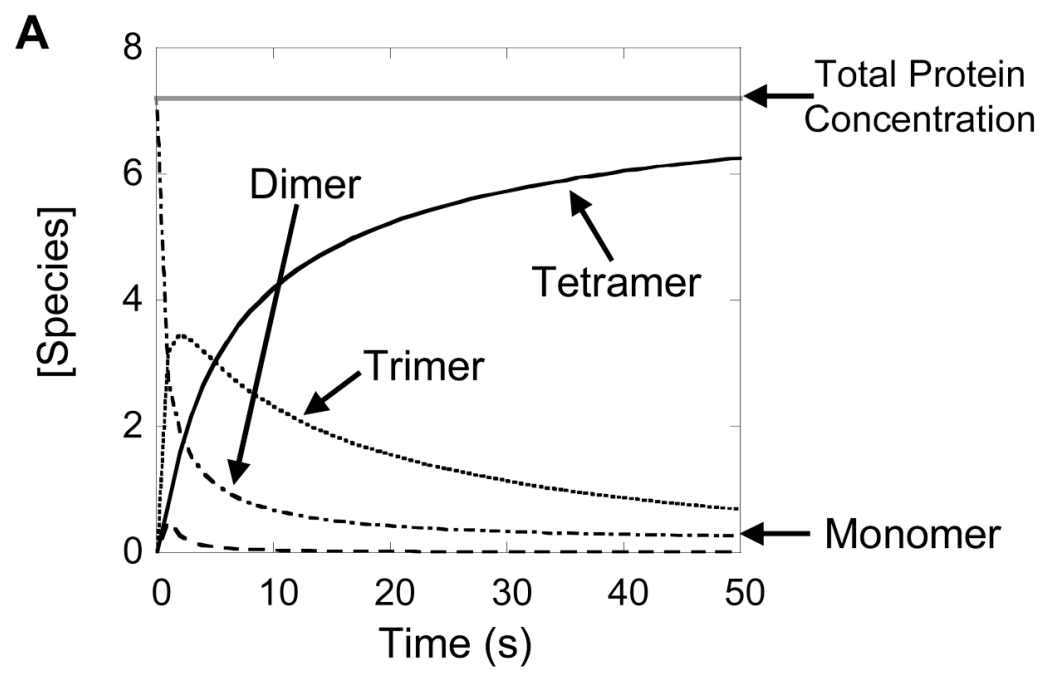

B

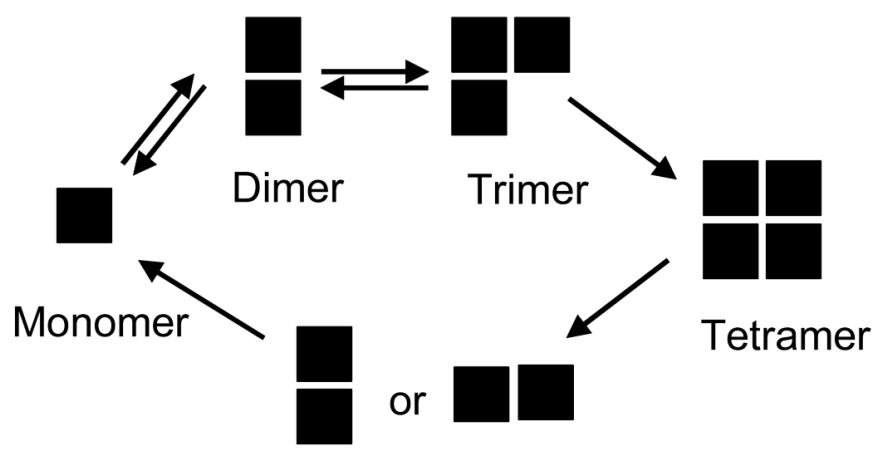

Dissociative Dimer

C

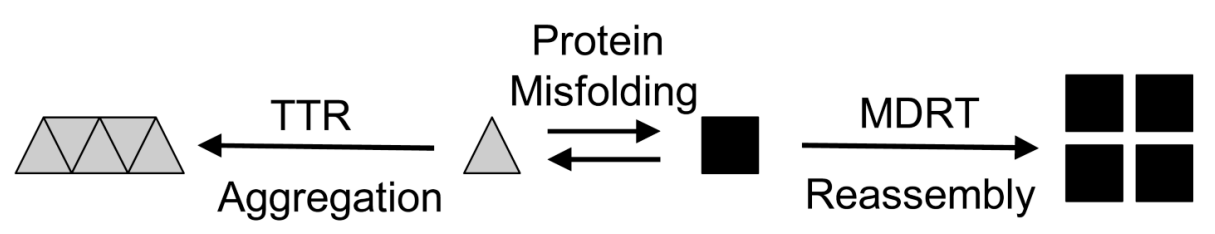

Figure 8.

Graphic representation of the kinetic partitioning of TTR monomers. A) Kinetic plot of the concentration of monomer (- - -), dimer $(-\bullet--)$, trimer $(\bullet \bullet \bullet)$, and tetramer (solid line) during the reassembly of TTR tetramer ([Protein $]_{\text {tot }}=7.2 \mu \mathrm{M}$ ) as predicted by the rate constants determined by the fitting analysis (Table 1C). The dotted gray line indicates the total protein concentration representing the predicted amplitude upon completion of the reassembly reaction $(7.2 \mu \mathrm{M})$. The initial rate of TTR tetramer formation is fast because of the high concentration of monomer in solution. As the reaction proceeds to completion, the rate of tetramer formation becomes slower because of the depleted concentration of TTR monomer in solution. B) TTR tetramers are formed by the sequential addition of monomers to dimeric and trimeric intermediates along the reassembly pathway. The tetramer dissociates in a cooperative unfolding pathway through a dimeric intermediate. The dimeric intermediate along the dissociative pathway may be the same unstable dimer formed in the reassembly mechanism, explaining the cooperative unfolding of this species under denaturing conditions. C) TTR monomers can kinetically partition between the reassembly pathway (black symbols) and the 
protein aggregation pathway (gray symbols). The two mechanistic pathways are buffered by a protein misfolding step. The partitioning of TTR into these two pathways can dictate the rate and extent of TTR aggregation, potentially affecting disease pathology. 
Wiseman et al.

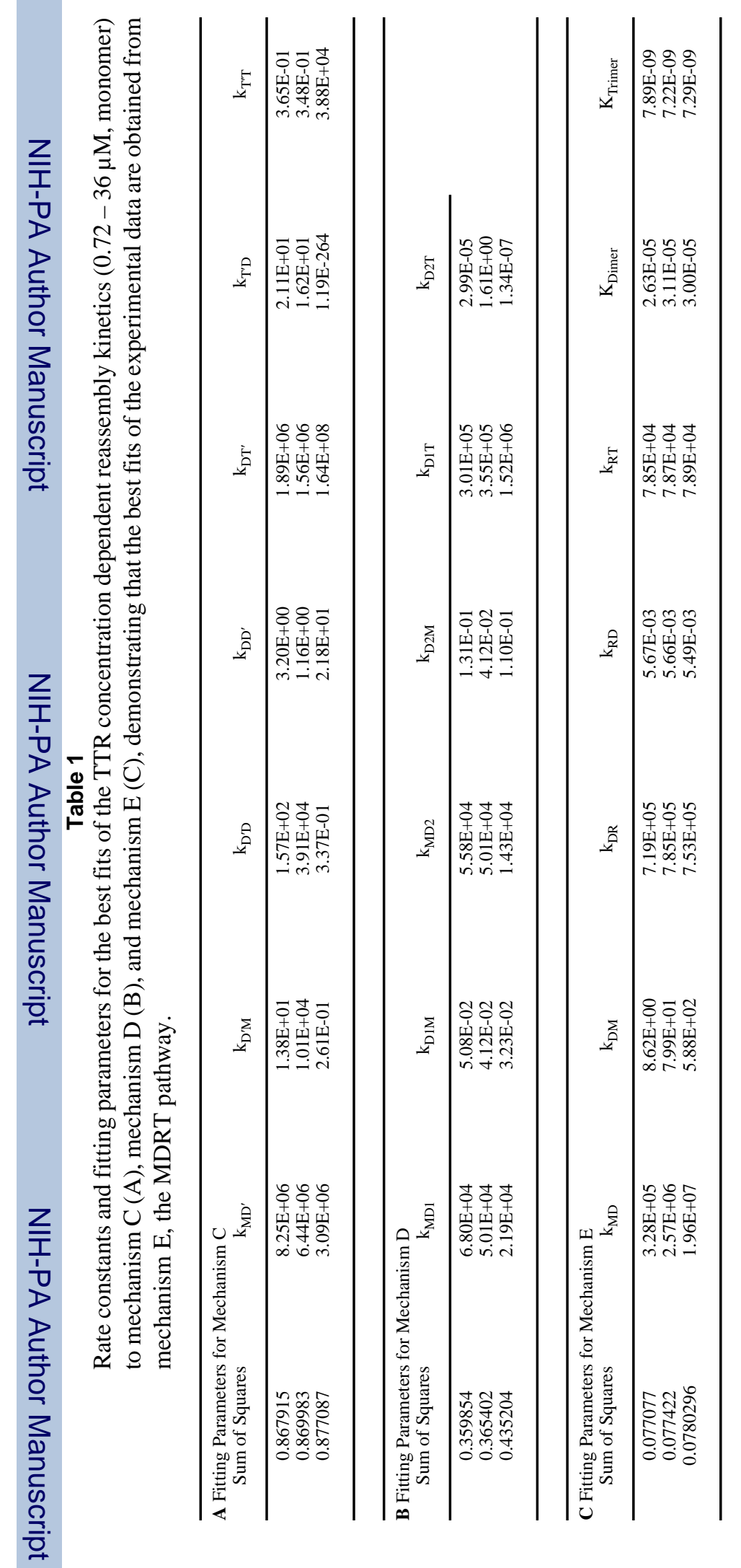

Biochemistry. Author manuscript; available in PMC 2008 September 9. 